\title{
Erratum to: Intraoperative Multispectral Fluorescence Imaging for the Detection of the Sentinel Lymph Node in Cervical Cancer: A Novel Concept
}

Lucia M. A. Crane, ${ }^{1}$ George Themelis, ${ }^{4}$ Rick G. Pleijhuis, ${ }^{1}$ Niels J. Harlaar, ${ }^{1,4}$ Athanasios Sarantopoulos, ${ }^{4}$ Henriette J. G. Arts, ${ }^{3}$ Ate G. J. van der Zee, ${ }^{3}$ Vasilis Ntziachristos, ${ }^{4}$ Gooitzen M. van Dam ${ }^{1,2}$

${ }^{1}$ Department of Surgery, University Medical Center Groningen, Groningen, the Netherlands

${ }^{2}$ Department of Surgery, BioOptical Imaging Center Groningen (BICG), University Medical Center Groningen, Hanzeplein 1, 9700 RB, Groningen, the Netherlands

${ }^{3}$ Department of Obstetrics and Gynaecology, University Medical Center Groningen, Groningen, the Netherlands

${ }^{4}$ Technical University Munich and Helmholtz Zentrum Munich, Institute for Biological and Medical Imaging (IBMI), Munich, Germany

T asilis Ntziachristos's name appeared incorrectly; it is correct as displayed here. 